\title{
Clinical Significance of C-Reactive Protein Levels in Predicting Responsiveness to Iron Therapy in Patients with Inflammatory Bowel Disease and Iron Deficiency Anemia
}

\author{
Tariq Iqbal • Jürgen Stein • Naveen Sharma • \\ Stefanie Kulnigg-Dabsch $\cdot$ Senthil Vel $\cdot$ Christoph Gasche
}

Received: 9 October 2014/ Accepted: 21 November 2014/Published online: 12 December 2014

(C) The Author(s) 2014. This article is published with open access at Springerlink.com

\begin{abstract}
Background Iron deficiency anemia (IDA) is a common complication of inflammatory bowel disease (IBD). In clinical practice, many patients receive initial treatment with iron tablets although intravenous (i.v.) iron supplementation is often preferable.

Aim This study investigated whether systemic inflammation at initiation of treatment (assessed by C-reactive protein [CRP] and interleukin-6 [IL-6] measurements) predicts response to iron therapy.
\end{abstract}

T. Iqbal $(\bowtie)$

Department of Gastroenterology, University Hospital Birmingham, Vincent Drive, Birmingham B15 2TH, UK e-mail: T.H.IQBAL@bham.ac.uk

\section{J. Stein}

Interdisciplinary Crohn Colitis Centre Rhein-Main, Schifferstrasse 59, 60594 Frankfurt am Main, Germany e-mail: j.stein@em.uni-frankfurt.de

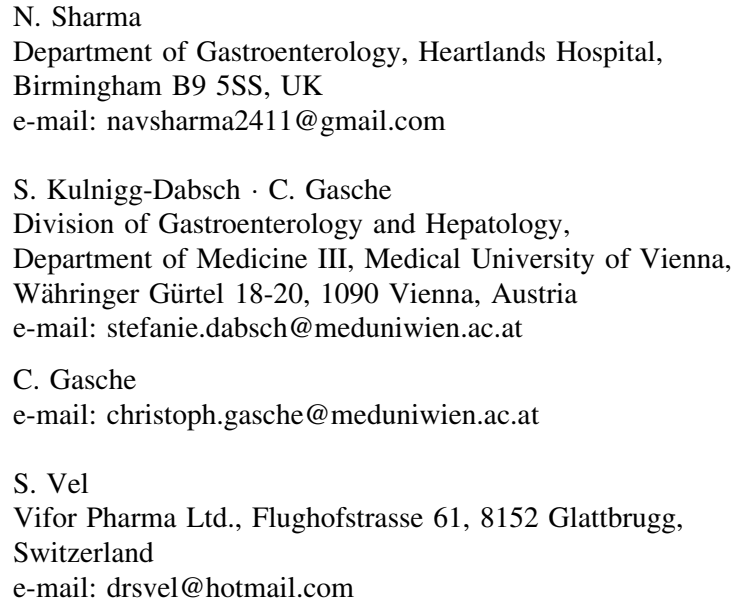

Methods Data from a previously published phase III trial were retrospectively analyzed after stratification of patients according to baseline CRP ( $>4$ vs. $\leq 4 \mathrm{mg} / \mathrm{L}$ ) and IL-6 (>6 vs. $\leq 6 \mathrm{pg} / \mathrm{mL}$ ) levels. The study population consisted of patients with Crohn's disease or ulcerative colitis and IDA $(\mathrm{Hb} \leq 110 \mathrm{~g} / \mathrm{L}$ and $\mathrm{TSAT}<20 \%$ or serum ferritin $<100 \mathrm{ng} / \mathrm{mL}$ ), randomized to either oral (ferrous sulfate) or i.v. iron (ferric carboxymaltose).

Results A total of 196 patients were evaluated (oral iron: $n=60$; i.v. iron: $n=136$ ). Baseline CRP and IL-6 levels were independent of patients' initial $\mathrm{Hb}$ levels and iron status (serum ferritin and TSAT; all $p>0.05$ ). Among iron tablet-treated patients, $\mathrm{Hb}$ increase was significantly smaller in the high- versus low-CRP subgroup (1.1 vs. 2.0, 2.3 vs. 3.1 , and 3.0 vs. $4.0 \mathrm{~g} / \mathrm{dL}$ at weeks 2 , 4 , and 8 , respectively; all $p<0.05)$. Differences were less pronounced with stratification according to baseline IL-6. Response to i.v. iron was mainly independent of inflammation.

Conclusions Patients with high baseline CRP achieved a lower $\mathrm{Hb}$ response with oral iron therapy. Our results suggest that CRP may be useful to identify IBD patients who can benefit from first-line treatment with i.v. iron to improve their IDA.

Keywords Crohn's disease - Ulcerative colitis . Inflammatory bowel disease $\cdot$ C-reactive protein . Iron deficiency · Iron therapy · Anemia

\section{Introduction}

Iron deficiency and iron deficiency anemia (IDA) are common complications in inflammatory bowel disease (IBD), IDA being a common cause of hospitalization and increased morbidity in this population $[1,2]$. Poor management of anemia can significantly affect the quality of 
life and lead to an increase in hospitalization rates and associated medical costs $[2,3]$.

The two most common causes of anemia in IBD patients are absolute iron deficiency and anemia of chronic disease (ACD; also referred to as anemia of chronic inflammation) $[1,3]$. Most IBD patients become iron deficient at some stages of the disease $[3,4]$. Causes of iron deficiency in IBD patients include intestinal bleeding, poor absorption of iron by the diseased intestine, and/or self-imposed dietary restrictions relating to gastrointestinal symptoms. ACD is driven by the systemic immune response that accompanies inflammatory disease such as IBD [5]. Cells of the immune system release pro-inflammatory cytokines, predominantly interleukin-6 (IL-6), which in turn up-regulate the expression of hepcidin, a key regulator of iron homeostasis. Hepcidin expression results in a reduction in the uptake of iron from the duodenum and a reduction in the ability of the body to utilize sufficient iron for effective erythropoiesis-also called functional iron deficiency $[5,6]$.

C-reactive protein (CRP) is widely used as a routine marker of chronic or acute inflammation [7]. Normal values for CRP range between 0.20 and $6.10 \mathrm{mg} / \mathrm{L}$ [8]. In clinical practice, CRP levels $\geq 5 \mathrm{mg} / \mathrm{L}$ are usually considered elevated. Following an inflammatory stimulus, causing, for example, IL-6 expression which can directly induce CRP expression in the liver, CRP levels rise rapidly to peak levels of $350-400 \mathrm{mg} / \mathrm{L}$. CRP is routinely measured in IBD patients and forms part of the recommended diagnostic workup in patients with anemia (hemoglobin $[\mathrm{Hb}]<12 \mathrm{~g} / \mathrm{dL}$ in non-pregnant women, $<13 \mathrm{~g} / \mathrm{dL}$ in men) $[3,9]$. A recent study in patients with IBD has shown a close correlation between CRP and hepcidin serum levels [10].

In addition to $\mathrm{Hb}$ and $\mathrm{CRP}$ measurements, the workup for IBD patients should include assessment of serum ferritin and transferrin saturation (TSAT) to accurately diagnose iron deficiency. Iron supplementation is recommended in all types of IBD-associated anemia where iron deficiency is present $[2,3,9]$. It has been proposed that chronic systemic inflammation may reduce the absorption of iron from the gut $[11,12]$. In addition, gut luminal iron may induce oxidative stress [13] and increase local disease activity in $\operatorname{IBD}[3,14]$. Nevertheless, in clinical practice a course of oral iron therapy is often used as first-line treatment for anemic patients with IBD.

This study aimed to investigate the effect of baseline systemic inflammation (as assessed by CRP and IL-6) on the treatment efficacy of iron supplementation comparing tablets to intravenous injections. The correlation between the degree of inflammation and response to oral or i.v. iron therapy was retrospectively analyzed in an IBD patient population from a previously reported phase III clinical trial [15].

\section{Materials and Methods}

Patient Population, Study Medication, and Study Design

Data from a randomized, multicenter, controlled phase III trial [15], which compared the efficacy and safety of i.v. ferric carboxymaltose (FCM) with oral ferrous sulfate (FS) in IBD patients, were retrospectively analyzed. The study design has been reported previously [15]. The study population consisted of patients with either $\mathrm{CD}$ or UC, who had iron deficiency anemia (defined by $\mathrm{Hb} \leq 11 \mathrm{~g} / \mathrm{dL}$ and TSAT $<20 \%$ or serum ferritin $<100 \mathrm{ng} / \mathrm{mL}$ ). Patients were randomized in a $2: 1$ ratio to receive either i.v. iron (FCM; max 1,000 mg iron per infusion at 1-week intervals until the patients' calculated total iron deficit was reached) or oral iron (FS; $100 \mathrm{mg}$ b.i.d. for 12 weeks) [15]. The study was conducted in accordance with good clinical practice guidelines and the Declaration of Helsinki, and was approved by the ethics committee at each site.

The predictive value of baseline CRP in terms of $\mathrm{Hb}$ response was assessed after retrospective stratification of the study population into a "high-CRP" and a "low-CRP" population. The median baseline CRP value of the study population was applied as the cutoff value. This prospective statistical approach resulted in a cutoff at $4 \mathrm{mg} / \mathrm{L}$ (high CRP: $>4 \mathrm{mg} / \mathrm{L}$; low CRP: $\leq 4 \mathrm{mg} / \mathrm{L}$ ) and ensured an equal number of patients in both groups. Similarly, the median baseline IL-6 value of the study population $(6 \mathrm{pg} / \mathrm{mL})$ was used for stratification into a "high-IL-6" ( $>6 \mathrm{pg} / \mathrm{mL})$ and "low-IL-6" $(\leq 6 \mathrm{pg} / \mathrm{mL})$ group.

\section{Laboratory Assessments/Outcome Measures Analyzed} for Correlation with Baseline Inflammatory Markers

Baseline measurements of CRP and IL-6 were available [15]. Outcome measures included $\mathrm{Hb}$ and iron status variables (serum ferritin and TSAT), with available assessments at baseline and weeks 2, 4, 8, and 12 [15].

\section{Statistical Analyses}

All analyses presented are consistent with the statistical methods used in the original clinical study report [15] and based on the full analysis set population (FAS). Data from all FAS patients with available baseline CRP were included for analysis. A small number of patients $(n=11)$ only had categorized CRP measurements that are available (e.g., $<5$, $<10 \mathrm{mg} / \mathrm{mL}$ ). These values were rounded down to the next integer. There was no imputation of missing data; analyses are based on the observed data. Baseline characteristic summary was described using counts for categorical variables and median and range for continuous variables. 
Correlations were estimated with Pearson's productmoment method. Group comparisons in $\mathrm{Hb}$ change from baseline and $\mathrm{Hb}$ standardized area under the curve (AUC) analysis were assessed using an analysis of covariance (ANCOVA) model with CRP or IL-6 group (high vs. low) as a factor and baseline $\mathrm{Hb}$ and gender as covariates. Statistical presentation includes least-squares means (LS means), standard errors (SE) split by baseline CRP or IL-6 (high vs. low) group, and associated level of significance. Statistical significance was assumed to be at $p<0.05$. Summary and statistical analyses were performed using SAS version 8 or later and $\mathrm{R}$ version 3.0.2.

\section{Results}

\section{Patient Characteristics}

All patients from the intention-to-treat population $(N=196)$ were evaluated for this retrospective analysis. Of these, 60 had received oral (CD: $n=16$; UC: $n=44$ ) and 136 i.v. iron (CD: $n=40$; UC: $n=96$ ).

Mean baseline $\mathrm{Hb}$ and laboratory measures of inflammatory markers were similar between treatment groups [15]. In the overall patient population, $46 \%$ had a baseline CRP level $\geq 5 \mathrm{mg} / \mathrm{L}$. CRP and IL-6 values were slightly higher in $\mathrm{CD}$ versus UC patients, but the differences were statistically not significant (Table 1).

\section{Inflammatory Markers in Relation to Baseline $\mathrm{Hb}$} and Iron Status

A potential relation of baseline CRP and IL-6 values with baseline $\mathrm{Hb}$ and iron status was investigated to assess whether these markers would be independent. For both markers, there was no significant relationship with baseline $\mathrm{Hb}$ levels (CRP: $R=0.0191, p=0.791$; IL-6: $R=$ $-0.0393, p=0.590)$. In addition, both markers proved to be independent of baseline TSAT (CRP: $R=-0.0337$, $p=0.657$; IL-6: $R=0.0708, p=0.358)$ and serum ferritin (CRP: $R=0.1313, p=0.074$; IL-6: $R=0.0443$, $p=0.554)$. Of note, baseline levels of CRP and IL-6

Table 1 Laboratory measures of inflammatory markers (full analysis set; median [range])

\begin{tabular}{llll}
\hline & $\begin{array}{l}\mathrm{CD} \\
(n=56)\end{array}$ & $\begin{array}{l}\mathrm{UC} \\
(n=140)\end{array}$ & $\begin{array}{l}\text { All patients } \\
(n=196)\end{array}$ \\
\hline $\mathrm{CRP}(\mathrm{mg} / \mathrm{L})$ & $7.4[0.0-66.8]$ & $3.5[0.0-71.4]$ & $4.0[0.0-71.4]$ \\
$\mathrm{IL}-6(\mathrm{pg} / \mathrm{mL})$ & $6.6[1.0-305.6]$ & $5.8[1.0-149.5]$ & $6.0[1.0-305.6]$
\end{tabular}

$C D$ Crohn's disease, $U C$ ulcerative colitis, $C R P$ C-reactive protein, IL-6 interleukin-6 strongly correlated with each other $(R=0.2442$; $p=0.0007)$.

\section{Correlation of Baseline CRP Levels with Hb Response}

Patients were stratified by baseline CRP levels into a "highCRP" ( $>4 \mathrm{mg} / \mathrm{L} ; n=96)$ and a "low-CRP" ( $\leq 4 \mathrm{mg} / \mathrm{L}$; $n=100)$ group, and responsiveness to iron supplementation (Hb change from baseline) was compared.

Among oral iron-treated patients, those with high baseline CRP had a significantly smaller mean $\mathrm{Hb}$ increase than those with low baseline CRP at follow-up visits on week 2, 4, and 8 ( $n=60$; Fig. 1a, $p<0.05$ ). Also at week 12, Hb increment tended to be smaller in the high- versus low-CRP group, but the difference did not reach statistical significance. Subpopulation analysis showed a significantly smaller $\mathrm{Hb}$ increase $(p<0.05)$ in the high- versus low-CRP group at weeks 2, 4, and 8 among UC patients treated with oral iron ( $n=44$; Fig. 1c). In CD patients who received oral iron $(n=16)$, early $\mathrm{Hb}$ increase was significantly smaller in the high-CRP group at week 2 (Fig. $1 \mathrm{~b}, p<0.05$ ) and tended to be smaller until week 8 compared to the low-CRP group. Standardized AUC analysis confirmed a statistically significant difference between the CRP groups in oral iron-treated patients (high vs. low CRP, LS means: 21.3 vs. 29.3; $p=0.012$ ). AUC was also statistically significantly different in the subgroup of UC patients (high vs. low CRP, LS means: 22.7 vs. $31.2 ; p=0.031$ ) but did not reach statistical significance in $\mathrm{CD}$ patients despite a large numerical difference (high vs. low CRP, LS means: 18.3 vs. 25.6; $p=0.250$ ).

Among i.v. iron-treated patients, there were no significant differences between the high- and low-CRP groups at weeks 1, 4, and 12 (Fig. 1d-f). A significant difference was observed in the overall population at week 8 only (Fig. 1d), and analysis of subpopulations showed that this difference was present in UC but not in CD patients (Fig. 1e, f). In line with these results, standardized AUC showed no statistically significant differences between high- and lowCRP groups in i.v. iron-treated patients (all patients, LS means: 26.3 vs. $29.7, p=0.105$; CD patients: 24.0 vs. $25.9, p=0.671$; UC patients: 27.7 vs. $30.8, p=0.189$ ).

Correlation of Baseline IL-6 Levels with Response

Patients were stratified by baseline IL-6 levels into a "highIL-6" ( $>6 \mathrm{pg} / \mathrm{mL} ; n=95)$ and a "low-IL-6" ( $\leq 6 \mathrm{pg} / \mathrm{mL}$; $n=95$ ) groups, and responsiveness to iron supplementation ( $\mathrm{Hb}$ change from baseline) was compared.

In oral iron-treated patients, a considerable trend toward lower $\mathrm{Hb}$ response was observed in the high- versus lowIL-6 groups, but differences did not quite reach the statistical significance $(p<0.07$ at weeks 2 and 8; Fig. 2a), 
A All patients, oral iron $(n=60)$

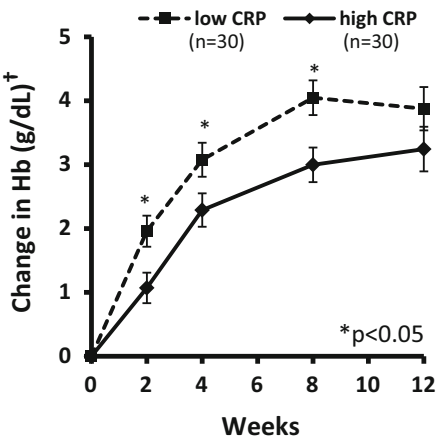

D All patients, i.v. iron $(n=136)$

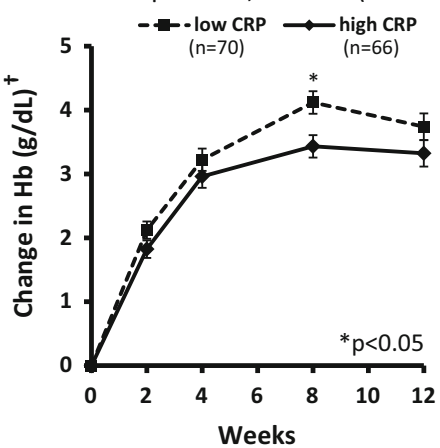

B Patients with $C D$, oral iron $(n=16)$

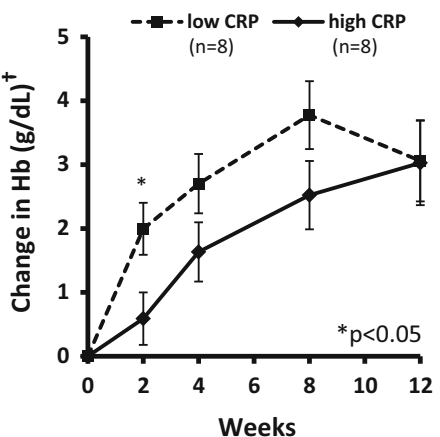

E Patients with $C D$, i.v. iron $(n=40)$

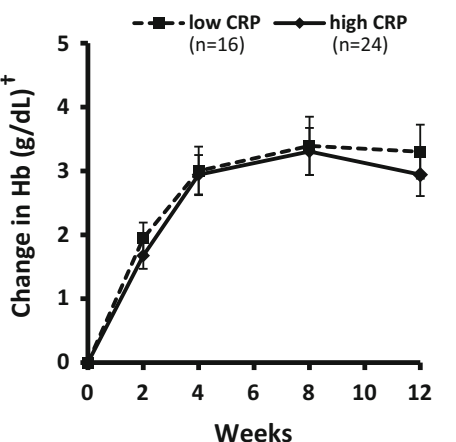

C Patients with UC, oral iron $(n=44)$

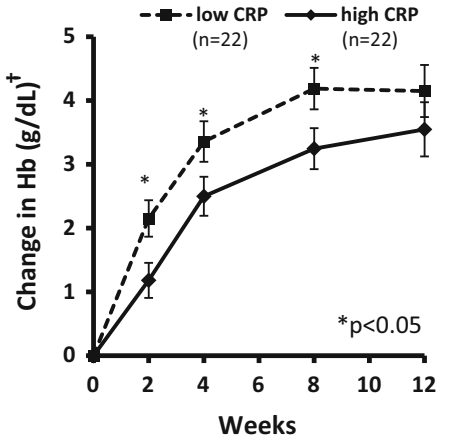

F Patients with UC, i.v. iron ( $n=96)$

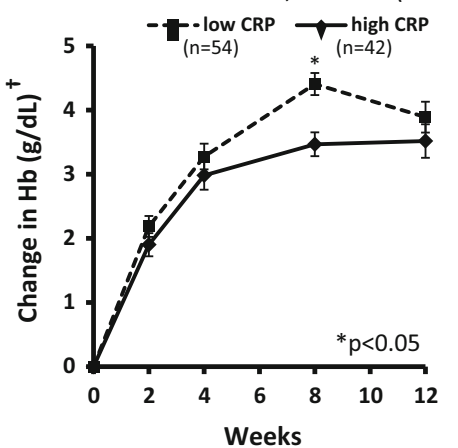

Fig. 1 Responsiveness of IBD patients with high versus low baseline CRP. ${ }^{\dagger}$ Least-squares means \pm standard errors, adjusted for gender and baseline Hb. $C D$ Crohn's disease, $C R P$ C-reactive protein, $H b$ hemoglobin, i.v. intravenous, $U C$ ulcerative colitis

apart from early response in the $\mathrm{CD}$ subpopulation $(p<0.05$ at week 2; Fig. 2b). In i.v. iron-treated patients, no significant differences between the high- and low-IL-6 groups were observed (overall population and $\mathrm{CD} / \mathrm{UC}$ subpopulations; Fig. 2d-f).

\section{Discussion}

This is the largest study showing an impact of systemic inflammation on iron absorption in anemic IBD patients. Initial $\mathrm{Hb}$ response to oral iron was significantly lower in IBD patients with a high baseline CRP level $(>4 \mathrm{mg} / \mathrm{L})$, compared to those with low baseline CRP-at least for the first 2 months of treatment. Hb response to i.v. iron was mainly independent of baseline CRP. The results of this study suggest that patients with IBD and IDA, who have elevated CRP at initiation of treatment, may benefit from first-line treatment with i.v. iron to improve their IDA and obtain a more rapid response.

Our current findings are in line with the established link between inflammation and iron sequestration. In the inflammatory state, increased hepcidin levels block duodenal iron absorption [16]. Hepcidin binds to the iron exporter ferroportin located on the basolateral surface of gut enterocytes and causes its internalization and degradation. In addition, high hepcidin levels also block the release of ferritin-bound iron from cells of the reticuloendothelial system (e.g., macrophages) [6, 17]. This hepcidin block during inflammation leads to diminished amounts of free iron available for erythropoiesis [5]. Recent data suggest that there are also other pro-inflammatory pathways modulating duodenal iron absorption. In mice treated with the inflammatory cytokine TNF- $\alpha$, reduced iron transport across the duodenal mucosa was shown [18, 19]. In patients with celiac disease, a correlation between iron deficiency and increased expression of ferritin in enterocytes of the duodenum was observed, indicating a link between iron absorption and local mucosal inflammation [20].

Our results are supported by the findings of three other studies. Significantly impaired oral iron absorption was reported in pediatric patients $(n=19)$ with active (defined by IL- $6>5 \mathrm{pg} / \mathrm{mL}$ ) versus inactive CD [11]. Serum iron levels inversely correlated with baseline CRP. As the test subjects were not anemic, only a single dose of oral iron was administered. The study lacked longer follow-up with prolonged iron administration and assessment of hematological response. Another study, a retrospective subanalysis from a phase III trial, found that a high baseline hepcidin 
A All patients, oral iron $(n=59)$

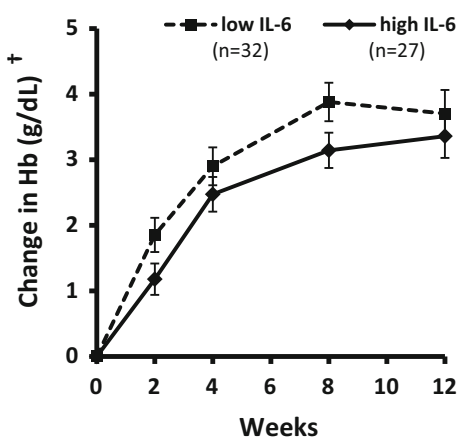

D All patients, i.v. iron $(n=131)$

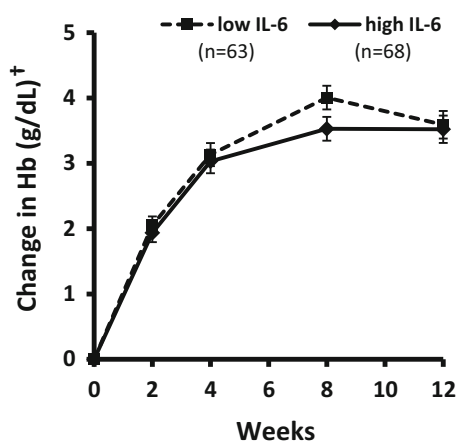

B Patients with $C D$, oral iron $(n=16)$

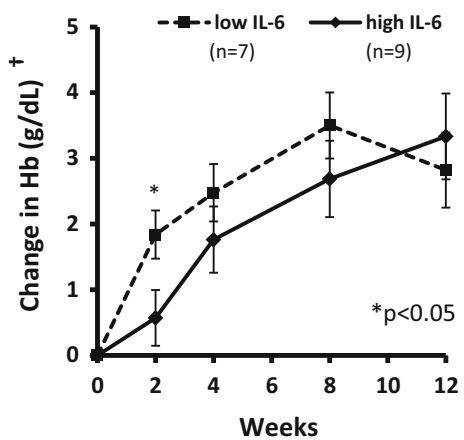

E Patients with $C D$, i.v. iron $(n=38)$

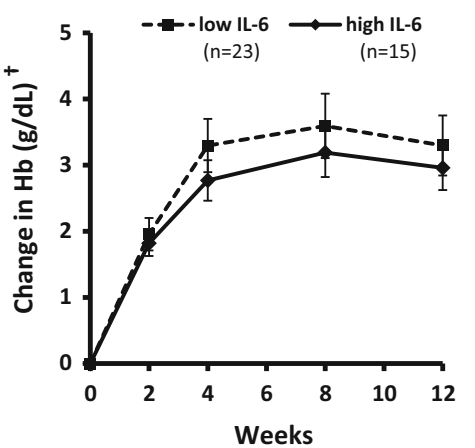

C Patients with UC, oral iron ( $n=43$ )

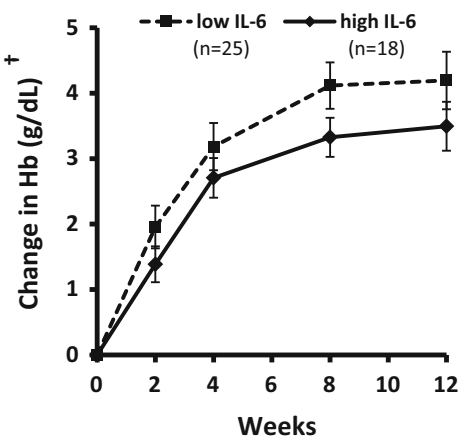

F Patients with UC, i.v. iron ( $n=93)$

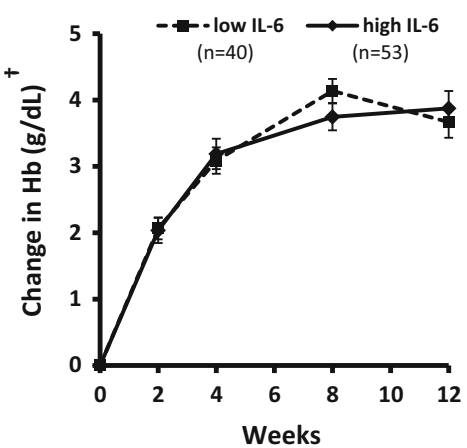

Fig. 2 Responsiveness of IBD patients with high versus low baseline IL-6. ${ }^{\dagger}$ Least-squares means \pm standard errors, adjusted for gender and baseline Hb. $C D$ Crohn`s disease, $H b$ hemoglobin, $I L-6$ interleukin-6, i.v. intravenous, $U C$ ulcerative colitis

level ( $>20 \mathrm{ng} / \mathrm{mL})$ could predict reduced responsiveness to oral iron in anemic patients with chronic kidney disease $(n=240)$ [21]. However, the potential of hepcidin as a biomarker is limited, even though it is a more direct measure of iron sequestration than the well-established but unspecific inflammatory marker CRP. Hepcidin immunoassays may detect inactive forms as well as the biologically active form, hepcidin-25, and measurements can vary up to tenfold between different assays, making it difficult to determine reference values [22]. Other analytical methods utilizing mass spectrometry are not feasible for routine use in a hospital or outpatient setting [23]. Lastly, a recently presented investigation from our group has shown that IBD patients with ACD respond differently to oral iron treatment compared to those with IDA [12]. IBD patients with ACD had impaired oral iron absorption which correlated with disease activity and inflammatory markers but was independent of disease location and type of IBD (UC or CD).

Intravenous iron can for the most part overcome the hepcidin block [24] and is therefore advisable in IBD patients with pronounced disease activity $[3,9]$. As shown in our and one other study [25], responsiveness to i.v. iron treatment appears to be independent of inflammatory status in IBD patients. Moreover, i.v. iron has been shown to be at least as effective as oral iron in randomized trials, delivering faster response rates and being better tolerated than oral iron [3, 9, 15, 26-28].

CRP is a readily available laboratory value that is usually included in the routine assessment of IBD patients. The cutoff level for stratification of patients into a highand a low-CRP subgroup was $4 \mathrm{mg} / \mathrm{L}$, based on the median CRP level in the evaluated population. This cutoff is very close to the $5 \mathrm{mg} / \mathrm{L}$ cutoff which is commonly used in clinical practice to identify the presence of inflammation. Notably, the median baseline CRP indicates that almost half of the patients had elevated levels of acute-phase proteins. This should also be considered in the selection of diagnostic markers for assessment of iron status. Ferritin is an acute-phase protein and its serum levels are elevated during inflammation, reflecting the inflammatory state rather than iron stores. Not surprisingly, a trend toward a positive correlation ( $p=0.075$ ) between baseline CRP and serum ferritin levels was observed in the study.

The results of this study are indicative of a significant biological effect of systemic inflammation on iron uptake, but patient numbers were relatively small. In particular, the small number of oral iron-treated CD patients did not allow for meaningful subanalyses, although differences are likely to be more pronounced in this subpopulation due to greater 
systemic inflammation compared to UC patients [7]. The relevance of a single significant difference between the high- and low-CRP groups among i.v. iron-treated patients (at week 8) needs to be evaluated. It may reflect delayed release of iron from reticuloendothelial stores in active disease. We also investigated baseline IL-6 levels in our study, and some significant differences between the highand low-IL-6 groups in terms of $\mathrm{Hb}$ response to oral iron could be observed. However, the predictive power of IL-6 was weak compared to CRP. The measurement of evaluated variables by local laboratories in this multicenter study may have added some additional variability into the data. Cytokines such as IL-6 are prone to degradation, and proper handling and storage of the samples are critical for the accuracy of the measurements. Published data suggest that IL-6 levels may be more relevant in CD than those in UC patients [29]. In future studies of this kind, measurement of hepcidin levels would be useful and medium-term follow-up of iron status establishes the mechanism of the effect. Of note, the efficacy data presented here are not confounded by compliance issues since adherence was monitored in the trial and was excellent $(99.2 \%)$ [15].

In conclusion, assessment of inflammatory status in terms of easily available CRP measurements could be useful in identifying patients who may initially benefit from first-line i.v. iron therapy. Based on the data from this relatively small patient group, patients with high CRP levels take longer to respond to oral iron but eventually reach similar responses at 3 months. Although the observed differences to the i.v. iron group are small, they may be important in clinical practice, where response to oral iron is often compromised by adherence problems. In the clinical trial setting, patients are selected for their willingness to participate and are more motivated to take oral iron, despite gastrointestinal side effects associated with this treatment. Overall, validation of the current findings in a larger patient sample is needed, including the investigation of potential differences in the predictive power for $\mathrm{CD}$ and UC patients, before clinical recommendations can be made.

Acknowledgments The authors acknowledge statistical support from Patrick Johnson (JPharma Solutions $\mathrm{GmbH}$ ) and medical writing support from Bettina Barton (SFL Regulatory Affairs \& Scientific Communication). Beate Rzychon (Vifor Pharma Ltd, Switzerland) reviewed and commented on the manuscript. Statistical support and medical writing support were funded by Vifor Pharma Ltd.

Conflict of interest TI received lecture fees from Vifor Pharma Ltd and Shire Pharmaceuticals Inc. JS has received consulting fees and been a member of advisory committees or review panels of Abbott Laboratories, Falk Pharma, Ferring Pharmaceuticals Inc, Fresenius Kabi Ltd, Immundiagnostik AG, MSD, Nycomed, Shire Pharmaceuticals Inc, and Vifor Pharma Ltd. NS has no conflicts of interest to disclose. SKD received speaker honoraria from Vifor International. SV is an employee of Vifor Pharma Ltd. CG has received grant and consultancy honoraria from Vifor Pharma Ltd, Pharmacosmos A/S, Fresenius Medical Care, and Renapharma Sweden.

Open Access This article is distributed under the terms of the Creative Commons Attribution Noncommercial License which permits any noncommercial use, distribution, and reproduction in any medium, provided the original author(s) and the source are credited.

\section{References}

1. Gisbert JP, Gomollon F. Common misconceptions in the diagnosis and management of anemia in inflammatory bowel disease. Am J Gastroenterol. 2008;103:1299-1307.

2. Stein J, Hartmann F, Dignass AU. Diagnosis and management of iron deficiency anemia in patients with IBD. Nat Rev Gastroenterol Hepatol.. 2010;7:599-610.

3. Gasche C, Berstad A, Befrits R, Beglinger C, et al. Guidelines on the diagnosis and management of iron deficiency and anemia in inflammatory bowel diseases. Inflamm Bowel Dis. 2007;13:1545-1553.

4. Kulnigg S, Gasche C. Systematic review: managing anaemia in Crohn's disease. Aliment Pharmacol Ther. 2006;24:1507-1523.

5. Weiss G, Goodnough LT. Anemia of chronic disease. $N$ Engl J Med. 2005;352:1011-1023.

6. Nemeth E, Tuttle MS, Powelson J, Vaughn MB, et al. Hepcidin regulates cellular iron efflux by binding to ferroportin and inducing its internalization. Science. 2004;306:2090-2093.

7. Vermeire S, Van AG, Rutgeerts P. Laboratory markers in IBD: useful, magic, or unnecessary toys? Gut. 2006;55:426-431.

8. Kindmark CO. The concentration of C-reactive protein in sera from healthy individuals. Scand J Clin Lab Invest. 1972;29:407-411.

9. Van Assche G, Dignass A, Bokemeyer B, Danese S, et al. Second European evidence-based consensus on the diagnosis and management of ulcerative colitis part 3: special situations. J Crohns Colitis.. 2013;7:1-33.

10. Wiesenthal M, Dignass A, Hartmann F, Iqbal T, Stein J. Serum hepcidin levels predict intestinal iron absorption in IBD patients. J Crohns Colitis.. 2014;8:S120.

11. Semrin G, Fishman DS, Bousvaros A, Zholudev A, et al. Impaired intestinal iron absorption in Crohn's disease correlates with disease activity and markers of inflammation. Inflamm Bowel Dis. 2006;12:1101-1106.

12. Wiesenthal M, Dignass A, Hartmann F, Stein J. The type of iron deficiency anaemia, but not the underlying disease, predicts intestinal iron absorption in IBD patients. J Crohns Colitis.. 2014;8:S195-S196.

13. Erichsen K, Hausken T, Ulvik RJ, Svardal A, Berstad A, Berge RK. Ferrous fumarate deteriorated plasma antioxidant status in patients with Crohn disease. Scand J Gastroenterol. 2003;38:543-548.

14. de Silva AD, Tsironi E, Feakins RM, Rampton DS. Efficacy and tolerability of oral iron therapy in inflammatory bowel disease: a prospective, comparative trial. Aliment Pharmacol Ther. 2005; 22:1097-1105.

15. Kulnigg S, Stoinov S, Simanenkov V, Dudar LV, et al. A novel intravenous iron formulation for treatment of anemia in inflammatory bowel disease: the ferric carboxymaltose (FERINJECT) randomized controlled trial. Am J Gastroenterol. 2008;103:1182-1192.

16. Evstatiev R, Gasche C. Iron sensing and signalling. Gut. 2012; 61:933-952.

17. Fillet G, Beguin Y, Baldelli L. Model of reticuloendothelial iron metabolism in humans: abnormal behavior in idiopathic hemochromatosis and in inflammation. Blood. 1989;74:844-851.

18. Sharma N, Laftah AH, Brookes MJ, Cooper B, Iqbal T, Tselepis C. A role for tumour necrosis factor alpha in human small bowel iron transport. Biochem J. 2005;390:437-446. 
19. Laftah AH, Sharma N, Brookes MJ, McKie AT, et al. Tumour necrosis factor alpha causes hypoferraemia and reduced intestinal iron absorption in mice. Biochem J. 2006;397:61-67.

20. Sharma N, Begum J, Eksteen B, Elagib A, et al. Differential ferritin expression is associated with iron deficiency in coeliac disease. Eur J Gastroenterol Hepatol. 2009;21:794-804.

21. Bregman DB, Morris D, Koch TA, He A, Goodnough LT. Hepcidin levels predict nonresponsiveness to oral iron therapy in patients with iron deficiency anemia. Am J Hematol. 2013;88:97-101.

22. Macdougall IC, Malyszko J, Hider RC, Bansal SS. Current status of the measurement of blood hepcidin levels in chronic kidney disease. Clin J Am Soc Nephrol.. 2010;5:1681-1689.

23. Ward DG, Roberts K, Stonelake P, Goon P, et al. SELDI-TOFMS determination of hepcidin in clinical samples using stable isotope labelled hepcidin as an internal standard. Proteome Sci.. 2008;6:28.

24. Aapro M, Osterborg A, Gascon P, Ludwig H, Beguin Y. Prevalence and management of cancer-related anaemia, iron deficiency and the specific role of intravenous iron. Ann Oncol. 2012;23:1954-1962.

25. Gasche C, Waldhoer T, Feichtenschlager T, Male C, et al. Prediction of response to iron sucrose in inflammatory bowel diseaseassociated anemia. Am J Gastroenterol. 2001;96:2382-2387.
26. Schroder O, Mickisch O, Seidler U, de WA, et al. Intravenous iron sucrose versus oral iron supplementation for the treatment of iron deficiency anemia in patients with inflammatory bowel disease-a randomized, controlled, open-label, multicenter study. Am J Gastroenterol. 2005; 100:2503-2509.

27. Lindgren $\mathrm{S}$, Wikman $\mathrm{O}$, Befrits $\mathrm{R}$, Blom $\mathrm{H}$, et al. Intravenous iron sucrose is superior to oral iron sulphate for correcting anaemia and restoring iron stores in IBD patients: a randomized, controlled, evaluator-blind, multicentre study. Scand J Gastroenterol. 2009;44:838-845.

28. Reinisch W, Staun M, Tandon RK, Altorjay I, et al. A randomized, open-label, non-inferiority study of intravenous iron isomaltoside 1,000 (Monofer) compared with oral iron for treatment of anemia in IBD (PROCEED). Am J Gastroenterol. 2013;108:1877-1888.

29. Hyams JS, Fitzgerald JE, Treem WR, Wyzga N, Kreutzer DL. Relationship of functional and antigenic interleukin 6 to disease activity in inflammatory bowel disease. Gastroenterology. 1993;104:1285-1292. 